\title{
Birnbaum criticality and importance measures for multistate systems with repairable components
}

\author{
Arne Bang Huseby \\ University of Oslo, Norway \\ Martyna Kalinowska \\ University of Oslo, Norway \\ Tobias Abrahamsen \\ University of Oslo, Norway
}

\begin{abstract}
We suggest four new measures of importance for repairable multistate systems based on the classical Birnbaum measure. Periodic component life cycles and general semiMarkov processes are considered. Similar to the Birnbaum measure the proposed measures are generic in the sense that they only depend on the probabilistic properties of the components and the system structure. The multistate system model encodes physical properties of the components and the system directly into the structure function. As a result, calculating importance is easy, especially in the asymptotic case. Moreover, the proposed measures are composite measures, combining importance for all component states into a unified quantity. This simplifies ranking of the components with respect to importance. The proposed measures can be characterised with respect to two features: forward-looking versus backward-looking, and with respect to how criticality is measured. Forward-looking importance measures focus on the next component states, while backward-looking importance measures focus on the previous component states. Two approaches to measuring criticality are considered: probability of criticality versus expected impact. Examples show that the different importance measures may result in unequal rankings.
\end{abstract}

\section{Component CRiticality; Birnbaum importance; Multistate systems; Repairable COMPonents; Semi-Markov processes}

\section{Introduction}

A main problem in reliability theory is to determine how the reliability of a complex system can be determined from knowledge of the reliabilities of its components. A weakness of traditional binary reliability theory is that the system and the components are always described just as functioning or failed. This approach often represents an oversimplification in real-life situations where the system and their components are capable of assuming a whole range of levels of performance, varying from perfect functioning to complete failure. In order to allow more detailed system models multistate systems were introduced in the mid 1980s. The methodology for such systems has been developed continuously since then. For an extensive introduction to multistate systems we refer to Natvig (2011b). 
The main topic of the present paper is importance measures for multistate systems. Recent reviews of papers on importance measures can be found in Hosseini et al. (2016) and Amrutkar and Kamalja (2017). There are two main reasons for calculating importance of components in a system. Firstly, it permits the analyst to determine which components merit the most additional research and development to improve overall system reliability at minimum cost or effort. Secondly, it may be used in diagnostics as a way of generating a list of components ordered with respect to how likely they are to have caused the system failure. In general no measure of importance can be expected to be universally best irrespective of usage purpose. Thus, comparing different measures is often of interest. Classical approaches to importance include Birnbaum (1969), Fussell and Vesely (1972), Barlow and Proschan (1975) and Natvig (1979). See also Natvig (1985) and Natvig and Gåsemyr (2009). All these classical measures can be described as generic in the sense that they depend only on the probabilistic properties of the components and the system structure.

One of the first papers on multistate systems is Griffith (1980). By studying how component improvement impacts the overall system reliability behavior, a notion of component importance was proposed. More specifically, the paper introduced a reliability importance vector where the $i$ th entry of the vector represented the impact on the system reliability given that the component was improved from state $i-1$ to state $i$. This approach can be viewed as a natural extension of the Birnbaum measure to the multistate case. A similar approach is essentially used in Natvig (2011a) and Natvig et al. (2011) for repairable systems.

For multistate systems with binary components Levitin and Lisnianski (1999) proposed sensitivity measures to quantify how much the system reliability is affected by perturbations in the components and in the stochastic system demand. Zio and Podofillini (2003) extended this to multistate systems with multistate components. This work was further developed in Levitin et al. (2003). See also Zio et al. (2004), Zio and Podofillini (2006) and Zio et al. (2007).

Reliability importance vectors contain a lot of useful information about how the components affect the system. In particular, for a given component, the most important component state with regard to its impact on system reliability can be identified. However, as pointed out by Ramirez-Marquez et al. (2006) the most critical system component state may not always correspond to the most critical system component. In order to simplify the comparison Ramirez-Marquez and Coit (2005) introduced composite importance measures which combine importance for all component states into a unified quantity. See also Ramirez-Marquez and Coit (2007). In Ramirez-Marquez et al. (2006) many different variations of composite importance measures are presented. A common idea behind these measures is that the importance of a component is quantified with respect to the impact on the system of fixing the component state at some level. Moreover, system performance is typically evaluated relative to the external demand the system is subject to. Both constant and variable demand models are considered.

Si et al. (2012) study integrated importance measure of component states for maintenance processes. To describe the impact of each component state in a maintenance process, a cost function of multistate systems is defined. Considering probability distributions, component transition rates, and system maintenance costs, the integrated importance measure of component states is described. The resulting characteristics are discussed in both series systems and parallel systems. See also Si et al. (2012). In Dui et al. (2015) this work is extended to general semi-Markov models. A similar framework is used in Dui et al. (2019) coupled with a general system model expressed in terms of multi-valued decision diagrams. 
In Dui et al. (2020) the proposed methodology is applied to a submarine blowout preventer system.

Si et al. (2013) extend the integrated importance measure to estimate the effect of a component residing at certain states on the performance of entire multistate systems. It is pointed out that many common importance measures do not consider how transition rates between different component states may affect the system. Thus, the authors present two new measures where these transition rates play a central role. Based on the first measure one may identify at which state a component should be kept in order to ensure a desired level of system performance. The second measure indicates which group of states one should keep the components at to provide the desired level of system performance and which component is the most important to keep at states above a certain level. It is shown that the state and component rankings according to the suggested importance measures depend on the transition rates of the components, on system structure and on the desired system performance.

Recent papers on importance measures take a decision oriented approach, where the importance measures are used as tools for optimizing performance and minimize cost. Papers in this category are e.g., Borgonovo and Apostolakis (2001), Wu and Coolen (2013), Wu et al. (2016), Cai et al. (2016), Skutlaberg and Natvig (2016), Zhu et al. (2017) and Cai et al. (2018). In order to facilitate this, the modelling framework must be extended to include additional elements such as objective functions, cost functions and demand models. This is indeed a very interesting development which makes importance measures applicable to a much wider range of problems.

Based on the cost-reliability relation, Si et al. (2019) proposes a generalized Birnbaum importance measure to quantify the contribution of individual components to system reliability improvement by considering reliability range, manufacturing complexity, and technology feasibility. The generalized measure can be applied to system reliability optimization.

In the present paper we focus on generic importance measures. We believe that such measures are especially useful in cases where knowledge about the operating environment and other external factors are unavailable or uncertain, e.g., in early design phases. At the same time, generic measures can often easily be put into a decision making context by including decisions and suitable objective functions.

In order to make component ranking straightforward, we have chosen to work with composite importance measures. While such measures are very easy to work with, there is a risk of oversimplifying the analysis. In particular, a single importance measure will never represent the full picture. In order to gain better insight into the strengths and weaknesses of a given system, it is often useful to apply more than one measure. Thus, in the present paper we propose four different generalizations of the classical Birnbaum measure. All these are true generalizations in the sense that they are reduced to the classical measure in the binary case. In the multistate case, however, the measures highlight different aspects of the system under consideration. The measures can be characterised along two axes: forward-looking versus backward-looking, and with respect to how criticality is measured. Forward-looking importance measures focus on the next component states. According to this approach the most important component is the one that has the highest probability of changing the system state. Backward-looking importance measures focus on the previous component states: According to this approach the most important component is the one that has the highest probability of having changed the system state. Furthermore, two approaches to measuring criticality are considered: probability of criticality versus impact 
of criticality.

We have chosen a modelling framework where the physical properties of the components and the system is encoded directly into the model. This eliminates the need for weight factors in the construction of the composite measures. Instead the impacts of the individual states are combined in a natural way without introducing extra parameters. As a result, calculating the importance measures can be done very efficiently, especially in the asymptotic case.

In the first sections of the paper each component follows periodic life cycles, starting out in the top state, and then transiting through the lower states until they fail. Then they are repaired or replaced, and a new life cycle starts. In the second part of the paper the life cycles of the components are modelled using general semi-Markov processes. The semi-Markov models used in this paper are similar to the ones considered in Dui et al. (2015).

\section{Multistate systems}

Before we introduce the importance measures, we briefly describe the modelling framework. We define a multistate system similar to a binary system as an ordered pair $(C, \phi)$, where $C=\{1, \ldots, n\}$ is the component set, and $\phi$ is the structure function. Moreover, we let $\boldsymbol{X}(t)=\left(X_{1}(t), \ldots, X_{n}(t)\right)$ where $X_{i}(t)$ is the state variable of component $i$ at time $t$. Contrary to a binary system, however, both the components and the system may be in multiple states (not just 0 and 1). More specifically, if $i \in C$, we let $S_{i}=\left\{0,1, \ldots, r_{i}\right\}$ denote the set of states for component $i$.

In the simplest cases we assume that each component starts out by being in its top-level state, i.e., state $r_{i}$ for component $i$. Then at random points of time the component transits downwards through the state set until it reaches state 0 . At this stage the component is repaired or replaced by a new component, and a new life cycle starts. In Section 4 we consider more general semi-Markov processes.

For each component $i \in C$ we also introduce a function $f_{i}: S_{i} \rightarrow \mathbb{R}$ representing the physical state of the component as a function of the state. Thus, if $X_{i}(t)=x_{i} \in S_{i}$, then the physical state of component $i$ at time $t$ is $f_{i}\left(X_{i}(t)\right)=f_{i}\left(x_{i}\right)$. If e.g., component $i$ is a pipeline, then the physical state of the component at a given point of time may be the capacity of the pipeline at this point of time. Being a physical property of the pipeline, this may be any non-negative number, and the function $f_{i}$ provides a convenient way of encoding this directly into the model.

Note that the functions $f_{1}, \ldots, f_{n}$ do not necessary need to be nondecreasing. By avoiding this restriction additional useful modelling flexibility is gained. This allows e.g., for the inclusion of burn-in phases, maintenance as well as minimal or partial repairs of a components as part of its life cycle before it reaches its failure state.

The structure function $\phi$ represents the state of the system expressed as a function of the states of the components. It is common in multistate reliability theory to assume that $\phi$ also assumes values in a set of non-negative integers. In this context, however, the structure function represents the physical state of the system. This function is expressed as a function of the physical states of the components:

$$
\phi(\boldsymbol{X}(t))=\phi\left(f_{1}\left(X_{1}(t)\right), \ldots, f_{n}\left(X_{n}(t)\right)\right)
$$

Thus, the physical state of the system is a function of the physical states of the components. 
The advantage with this approach is that the system state is expressed in terms of physical quantities rather than being encoded more abstractly as non-negative integers.

In the following we will also use the standard notation from reliability theory, $\phi\left(x_{i}, \boldsymbol{X}\right)$ defined as:

$$
\phi\left(x_{i}, \boldsymbol{X}\right)=\phi\left(X_{1}, \ldots, X_{i-1}, x_{i}, X_{i+1}, \ldots, X_{n}\right) .
$$

In cases where the index $i$ is obvious from the context, we simply write $\phi(x, \boldsymbol{X})$.

\section{Criticality and importance in multistate systems}

In order to extend the definition of criticality and importance we consider a multistate system $(C, \phi)$. Let $i \in C$, and let $S_{i}=\left\{0,1, \ldots, r_{i}\right\}$ be the set of states for this component. We then introduce $X_{i}^{+}(t)$ and $X_{i}^{-}(t)$ as respectively the next and previous state of component $i, i=1, \ldots, n$. With more than two possible states for each component, the mathematical expressions for $X_{i}^{+}(t)$ and $X_{i}^{-}(t)$ are different. More specifically, we have:

$$
\begin{aligned}
& X_{i}^{+}(t)= \begin{cases}X_{i}(t)-1 & \text { for } X_{i}(t)>0 \\
r_{i} & \text { for } X_{i}(t)=0\end{cases} \\
& X_{i}^{-}(t)= \begin{cases}X_{i}(t)+1 & \text { for } X_{i}(t)<r_{i} \\
0 & \text { for } X_{i}(t)=r_{i}\end{cases}
\end{aligned}
$$

Based on $X_{i}^{+}(t)$ and $X_{i}^{-}(t)$ we introduce two notions of criticality. We say that component $i$ is $n$-critical at time $t$ if:

$$
\phi\left(X_{i}(t), \boldsymbol{X}(t)\right) \neq \phi\left(X_{i}^{+}(t), \boldsymbol{X}(t)\right) .
$$

Hence, component $i$ is n-critical at time $t$ if changing the component to its next state would result in a system state change as well. Similarly we say that component $i$ is $p$-critical at time $t$ if:

$$
\phi\left(X_{i}^{-}(t), \boldsymbol{X}(t)\right) \neq \phi\left(X_{i}(t), \boldsymbol{X}(t)\right) .
$$

Hence, component $i$ is p-critical at time $t$ if changing the component back to its previous state would result in a system state change as well.

We then proceed by introducing two possible generalisations of the Birnbaum measure to multistate systems. We define the $n$-Birnbaum measure of importance of component $i$ at time $t$, denoted $I_{N B}^{(i)}(t)$, as the probability that the component is n-critical at time $t$. Similarly, we define the $p$-Birnbaum measure of importance of component $i$ at time $t$, denoted $I_{P B}^{(i)}(t)$, as the probability that the component is p-critical at time $t$. Hence, using Eq. (3.1) and Eq. (3.2) we get:

$$
\begin{aligned}
& I_{N B}^{(i)}(t)=P\left[\phi\left(X_{i}(t), \boldsymbol{X}(t)\right) \neq \phi\left(X_{i}^{+}(t), \boldsymbol{X}(t)\right)\right], \\
& I_{P B}^{(i)}(t)=P\left[\phi\left(X_{i}^{-}(t), \boldsymbol{X}(t)\right) \neq \phi\left(X_{i}(t), \boldsymbol{X}(t)\right)\right] .
\end{aligned}
$$

We observe that $I_{N B}^{(i)}(t)$ is the probability that the system state will change if component $i$ enters its next state, while $I_{P B}^{(i)}(t)$ is the probability that the system state changed as a result of the most recent state change for component $i$. Thus, $I_{N B}^{(i)}(t)$ is forward-looking, while $I_{P B}^{(i)}(t)$ is backward-looking. 
It is easy to verify that in in the binary case $I_{N B}^{(i)}(t)=I_{P B}^{(i)}(t)=I_{B}^{(i)}(t)$, where $I_{B}^{(i)}(t)$ denotes the well-known Birnbaum measure introduced by Birnbaum (1969). In the multistate case, however, we may have $I_{N B}^{(i)}(t) \neq I_{P B}^{(i)}(t)$. In order to take a closer look at the difference between the two importance measures, we rewrite the expressions Eq. (3.3) and Eq. (3.4) by conditioning on the state of component $i$ and get:

$$
\begin{aligned}
I_{N B}^{(i)}(t) & =\sum_{u=1}^{r_{i}} P[\phi(u, \boldsymbol{X}(t)) \neq \phi(u-1, \boldsymbol{X}(t))] \cdot P\left[X_{i}(t)=u\right] \\
& +P\left[\phi(0, \boldsymbol{X}(t)) \neq \phi\left(r_{i}, \boldsymbol{X}(t)\right)\right] \cdot P\left[X_{i}(t)=0\right] \\
I_{P B}^{(i)}(t) & =\sum_{u=0}^{r_{i}-1} P[\phi(u+1, \boldsymbol{X}(t)) \neq \phi(u, \boldsymbol{X}(t))] \cdot P\left[X_{i}(t)=u\right] \\
& +P\left[\phi(0, \boldsymbol{X}(t)) \neq \phi\left(r_{i}, \boldsymbol{X}(t)\right)\right] \cdot P\left[X_{i}(t)=r_{i}\right]
\end{aligned}
$$

Changing the summation index in Eq. (3.6) this expression becomes:

$$
\begin{aligned}
I_{P B}^{(i)}(t) & =\sum_{u=1}^{r_{i}} P[\phi(u, \boldsymbol{X}(t)) \neq \phi(u-1, \boldsymbol{X}(t))] \cdot P\left[X_{i}(t)=u-1\right] \\
& +P\left[\phi(0, \boldsymbol{X}(t)) \neq \phi\left(r_{i}, \boldsymbol{X}(t)\right)\right] \cdot P\left[X_{i}(t)=r_{i}\right]
\end{aligned}
$$

Comparing Eq. (3.5) and Eq. (3.7) we observe that $P\left[X_{i}(t)=u\right]$ in Eq. (3.5) is replaced by $P\left[X_{i}(t)=u-1\right]$ in Eq. (3.7), $u=1, \ldots, r_{i}$. Moreover, $P\left[X_{i}(t)=0\right]$ in Eq. (3.5) is replaced by $P\left[X_{i}(t)=r_{i}\right]$ in Eq. (3.7). From this it follows that if $P\left[X_{i}(t)=0\right]=P\left[X_{i}(t)=1\right]=$ $\cdots=P\left[X_{i}(t)=r_{i}\right]$, we will have $I_{N B}^{(i)}(t)=I_{P B}^{(i)}(t)$. In general, however, the two importance measures will be different.

Example 3.1 We consider a multistate system $(C, \phi)$ where $C=\{1,2\}$. Both components have three possible states, 0,1,2. For simplicity, we assume that these states are identical to the physical states, i.e., $f_{i}(u)=u, u=0,1,2$ and $i=1,2$. Moreover, the structure function is given by:

$$
\phi\left(X_{1}(t), X_{2}(t)\right)=\min \left(f_{1}\left(X_{1}(t)\right), f_{2}\left(X_{2}(t)\right)\right) .
$$

For a given $t$ we assume that $P\left[X_{1}(t)=u\right]=p_{u}$ and $P\left[X_{2}(t)=u\right]=q_{u}, u=0,1,2$. It is then easy to see that:

$$
\begin{aligned}
P\left[\phi\left(0, X_{2}(t)\right) \neq \phi\left(2, X_{2}(t)\right)\right] & =q_{1}+q_{2}, \\
P\left[\phi\left(1, X_{2}(t)\right) \neq \phi\left(0, X_{2}(t)\right)\right] & =q_{1}+q_{2}, \\
P\left[\phi\left(2, X_{2}(t)\right) \neq \phi\left(1, X_{2}(t)\right)\right] & =q_{2}, \\
P\left[\phi\left(X_{1}(t), 0\right) \neq \phi\left(X_{1}(t), 2\right)\right] & =p_{1}+p_{2}, \\
P\left[\phi\left(X_{1}(t), 1\right) \neq \phi\left(X_{1}(t), 0\right)\right] & =p_{1}+p_{2}, \\
P\left[\phi\left(X_{1}(t), 2\right) \neq \phi\left(X_{1}(t), 1\right)\right] & =p_{2} .
\end{aligned}
$$


Inserting this into Eq.(3.5) and Eq.(3.7) we get after some simplifications that:

$$
\begin{aligned}
& I_{N B}^{(1)}(t)=q_{1}+q_{2}-p_{2} q_{1}, \\
& I_{N B}^{(2)}(t)=p_{1}+p_{2}-p_{1} q_{2}, \\
& I_{P B}^{(1)}(t)=q_{1}+q_{2}-p_{1} q_{1}, \\
& I_{P B}^{(2)}(t)=p_{1}+p_{2}-p_{1} q_{1} .
\end{aligned}
$$

We observe that $I_{P B}^{(1)}(t)>I_{P B}^{(2)}(t)$ if and only if $q_{1}+q_{2}>p_{1}+p_{2}$. However, assuming that $q_{1}+q_{2}>p_{1}+p_{2}$ and at the same time choosing $p_{1}<p_{2}$ and $q_{1}>q_{2}$, it is possible to obtain the opposite ranking with respect to the $n$-Birnbaum measure.

Assume e.g., that $p_{1}=0.20, p_{2}=0.35, q_{1}=0.40$ and $q_{2}=0.20$. We then get $I_{N B}^{(1)}(t)=$ 0.46 while $I_{N B}^{(2)}(t)=0.51$. Furthermore, $I_{P B}^{(1)}(t)=0.52$ while $I_{P B}^{(2)}(t)=0.47$. That is, $I_{N B}^{(1)}(t)<I_{N B}^{(2)}(t)$ while $I_{P B}^{(1)}(t)>I_{P B}^{(2)}(t)$

Example 3.2 In this example we also consider a multistate system $(C, \phi)$ where $C=\{1,2\}$, and where both components have three possible states, 0,1,2. As in the previous example we let $f_{1}(u)=u, u=0,1,2$. However, in this case we let $f_{2}(0)=0, f_{2}(1)=2$ and $f_{2}(2)=1$. Thus, component 2 starts out in its intermediate physical state 1 before its best physical state 2 is reached. The structure function is once again:

$$
\phi\left(X_{1}(t), X_{2}(t)\right)=\min \left(f_{1}\left(X_{1}(t)\right), f_{2}\left(X_{2}(t)\right)\right),
$$

and for a given $t$ we let $P\left[X_{1}(t)=u\right]=p_{u}$ and $P\left[X_{2}(t)=u\right]=q_{u}, u=0,1,2$. We now get:

$$
\begin{aligned}
& P\left[\phi\left(0, X_{2}(t)\right) \neq \phi\left(2, X_{2}(t)\right)\right]=q_{1}+q_{2}, \\
& P\left[\phi\left(1, X_{2}(t)\right) \neq \phi\left(0, X_{2}(t)\right)\right]=q_{1}+q_{2}, \\
& P\left[\phi\left(2, X_{2}(t)\right) \neq \phi\left(1, X_{2}(t)\right)\right]=q_{1}, \\
& P\left[\phi\left(X_{1}(t), 0\right) \neq \phi\left(X_{1}(t), 2\right)\right]=p_{1}+p_{2}, \\
& P\left[\phi\left(X_{1}(t), 1\right) \neq \phi\left(X_{1}(t), 0\right)\right]=p_{1}+p_{2}, \\
& P\left[\phi\left(X_{1}(t), 2\right) \neq \phi\left(X_{1}(t), 1\right)\right]=p_{2} .
\end{aligned}
$$

Inserting this into Eq. (3.5) and Eq. (3.7) we get:

$$
\begin{aligned}
& I_{N B}^{(1)}(t)=q_{1}+q_{2}-p_{2} q_{2}, \\
& I_{N B}^{(2)}(t)=p_{1}+p_{2}-p_{1} q_{2}, \\
& I_{P B}^{(1)}(t)=q_{1}+q_{2}-p_{1} q_{2}, \\
& I_{P B}^{(2)}(t)=p_{1}+p_{2}-p_{1} q_{1} .
\end{aligned}
$$

We now assume that $P\left[f_{1}\left(X_{1}(t)\right)=u\right]=P\left[f_{2}\left(X_{2}(t)\right)=u\right], u=0,1,2$. This implies that $p_{2}=q_{1}$ and $p_{1}=q_{2}$. Focussing first on the $n$-Birnbaum measure, it follows that:

$$
I_{N B}^{(1)}(t)>I_{N B}^{(2)}(t) \quad \text { if and only if } p_{2}<p_{1} .
$$


Assume more specifically that $p_{2}=q_{1}=0.3$ and $p_{1}=q_{2}=0.4$. We then get:

$$
I_{N B}^{(1)}(t)=0.58 \quad \text { and } \quad I_{N B}^{(2)}(t)=0.54 .
$$

Thus, even though $f_{1}\left(X_{1}(t)\right)$ and $f_{2}\left(X_{2}(t)\right)$ have the same distribution, and the structure function is symmetric with respect to $f_{1}$ and $f_{2}$, component 1 has higher importance than component 2 when the $n$-Birnbaum measure is used. If instead $p_{2}>p_{1}$, the ranking is reversed. Similar results hold for the p-Birnbaum measure

\subsection{Importance based on expected physical criticality}

The importance measures introduced so far are based on a binary notion of criticality: either a component is critical, or it is not critical. At the same time our definition of the structure function $\phi$ is assumed to represent some physical quantity. In this section we utilise this as a basis for two new importance measures. More specifically, for a given multistate system $(C, \phi)$ we introduce $\Delta_{N}^{(i)}(t)$ and $\Delta_{P}^{(i)}(t)$ defined for $t>0$ and $i \in C$ as:

$$
\begin{aligned}
& \Delta_{N}^{(i)}(t)=\left|\phi\left(X_{i}(t), \boldsymbol{X}(t)\right)-\phi\left(X_{i}^{+}(t), \boldsymbol{X}(t)\right)\right| \\
& \Delta_{P}^{(i)}(t)=\left|\phi\left(X_{i}(t)^{-}, \boldsymbol{X}(t)\right)-\phi\left(X_{i}(t), \boldsymbol{X}(t)\right)\right|
\end{aligned}
$$

Hence, $\Delta_{N}^{(i)}(t)$ denotes the absolute value of the change in system state as a result of component $i$ changing from its current state $X_{i}(t)$ to its next state $X_{i}^{+}(t)$. Similarly, $\Delta_{P}^{(i)}(t)$ denotes the absolute value of the change in system state as a result of component $i$ changing from its previous state $X_{i}^{-}(t)$ to its current state $X_{i}(t)$.

The $n^{*}$-Birnbaum measure of importance of component $i$ at time $t$, denoted $I_{N B}^{*(i)}(t)$, is defined as the expected value of $\Delta_{N}^{(i)}(t)$ :

$$
I_{N B}^{*(i)}(t)=E\left|\phi\left(X_{i}(t), \boldsymbol{X}(t)\right)-\phi\left(X_{i}^{+}(t), \boldsymbol{X}(t)\right)\right| .
$$

Similarly, the $p^{*}$-Birnbaum measure of importance of component $i$ at time $t$, denoted $I_{N B}^{*(i)}(t)$, is defined as the expected value of $\Delta_{P}^{(i)}(t)$ :

$$
I_{P B}^{*(i)}(t)=E\left|\phi\left(X_{i}^{-}(t), \boldsymbol{X}(t)\right)-\phi\left(X_{i}(t), \boldsymbol{X}(t)\right)\right| .
$$

We observe that $I_{N B}^{*(i)}(t)$ is the expected physical impact on the system given that component $i$ enters its next state, while $I_{P B}^{*(i)}(t)$ is the expected impact of the most recent state change for component $i$. Thus, $I_{N B}^{*(i)}(t)$ is forward-looking, while $I_{P B}^{*(i)}(t)$ is backward-looking. The main difference between these measures and the previously introduced measures is in the way we quantify criticality. Expected physical impact can potentially capture more detailed information about the system than a simple probability.

Note that in the binary case all the different measures are the same:

$$
I_{N B}^{(i)}(t)=I_{P B}^{(i)}(t)=I_{N B}^{*(i)}(t)=I_{P B}^{*(i)}(t)=I_{B}^{(i)}(t) .
$$


In order to show how these measures can be computed, we expand the expressions Eq. (3.8) and Eq. (3.9) by conditioning on the state of component $i$ and get:

$$
\begin{aligned}
I_{N B}^{*(i)}(t) & =\sum_{u=1}^{r_{i}} E|\phi(u, \boldsymbol{X}(t))-\phi(u-1, \boldsymbol{X}(t))| \cdot P\left[X_{i}(t)=u\right] \\
& +E\left|\phi(0, \boldsymbol{X}(t))-\phi\left(r_{i}, \boldsymbol{X}(t)\right)\right| \cdot P\left[X_{i}(t)=0\right] \\
I_{P B}^{*(i)}(t) & =\sum_{u=0}^{r_{i}-1} E|\phi(u+1, \boldsymbol{X}(t))-\phi(u, \boldsymbol{X}(t))| \cdot P\left[X_{i}(t)=u\right] \\
& +E\left|\phi(0, \boldsymbol{X}(t))-\phi\left(r_{i}, \boldsymbol{X}(t)\right)\right| \cdot P\left[X_{i}(t)=r_{i}\right]
\end{aligned}
$$

Again we change the summation index in Eq. (3.11) and get:

$$
\begin{aligned}
I_{P B}^{*(i)}(t) & =\sum_{u=1}^{r_{i}} E|\phi(u, \boldsymbol{X}(t))-\phi(u-1, \boldsymbol{X}(t))| \cdot P\left[X_{i}(t)=u-1\right] \\
& +E\left|\phi(0, \boldsymbol{X}(t))-\phi\left(r_{i}, \boldsymbol{X}(t)\right)\right| \cdot P\left[X_{i}(t)=r_{i}\right]
\end{aligned}
$$

Using the same arguments as in the previous section, it follows that $I_{N B}^{*(i)}(t)=I_{P B}^{*(i)}(t)$ if $P\left[X_{i}(t)=0\right]=P\left[X_{i}(t)=1\right]=\cdots=P\left[X_{i}(t)=r_{i}\right]$. In general, however, the two importance measures will be different.

Example 3.3 Consider a multistate system $(C, \phi)$ where $C=\{1,2\}$, and where $S_{1}=\{0,1\}$ and $S_{2}=\{0,1,2\}$. Moreover, we assume that:

$$
\begin{aligned}
& f_{1}(u)=2 u, \quad u \in S_{1}, \\
& f_{2}(u)=u, \quad u \in S_{2} .
\end{aligned}
$$

As before, the structure function is given by:

$$
\phi\left(X_{1}(t), X_{2}(t)\right)=\min \left(f_{1}\left(X_{1}(t)\right), f_{2}\left(X_{2}(t)\right)\right) .
$$

Finally, we again assume that the component state processes are independent, and that for a given $t$ we have:

$$
\begin{aligned}
& P\left[X_{1}(t)=u\right]=p_{u}>0, \quad u \in S_{1}, \\
& P\left[X_{2}(t)=u\right]=q_{u}>0, \quad u \in S_{2} .
\end{aligned}
$$

Noting that component 1 only has two states, we get:

$$
E\left|\phi\left(1, X_{2}(t)\right)-\phi\left(0, X_{2}(t)\right)\right|=E\left|\phi\left(0, X_{2}(t)\right)-\phi\left(1, X_{2}(t)\right)\right|=q_{1}+2 q_{2}
$$

Inserting this into Eq. (3.10) and using that $p_{0}+p_{1}=1$ we get:

$$
I_{N B}^{*(1)}(t)=\left(q_{1}+2 q_{2}\right) p_{1}+\left(q_{1}+2 q_{2}\right) p_{0}=q_{1}+2 q_{2}
$$

For component 2 we have:

$$
\begin{aligned}
& E\left|\phi\left(X_{1}(t), 1\right)-\phi\left(X_{1}(t), 0\right)\right|=p_{1} \\
& E\left|\phi\left(X_{1}(t), 2\right)-\phi\left(X_{1}(t), 1\right)\right|=p_{1} \\
& E\left|\phi\left(X_{1}(t), 0\right)-\phi\left(X_{1}(t), 2\right)\right|=2 p_{1}
\end{aligned}
$$


Inserting this into Eq. (3.10) and using that $q_{0}+q_{1}+q_{2}=1$ we get:

$$
I_{N B}^{*(2)}(t)=p_{1} q_{1}+p_{1} q_{2}+2 p_{1} q_{0}=\left(1+q_{0}\right) p_{1}
$$

We also have:

$$
\begin{aligned}
& E\left[f_{1}\left(X_{1}(t)\right)\right]=0 \cdot p_{0}+2 \cdot p_{1}=2 p_{1} \\
& E\left[f_{2}\left(X_{2}(t)\right)\right]=0 \cdot q_{0}+1 \cdot q_{1}+2 \cdot q_{2}=q_{1}+2 q_{2} .
\end{aligned}
$$

In order to make the comparison between the components as fair as possible, we assume that their distributions are so that $E\left[f_{1}\left(X_{1}(t)\right)\right]=E\left[f_{2}\left(X_{2}(t)\right)\right]$, i.e., $2 p_{1}=q_{1}+2 q_{2}$. This implies that:

$$
\begin{aligned}
I_{N B}^{*(1)}(t) & =2 p_{1} \\
I_{N B}^{*(2)}(t) & =\left(1+q_{0}\right) p_{1}
\end{aligned}
$$

Since we have assumed that $q_{1}>0$ and $q_{2}>0$, it follows that $q_{0}<1$. Hence, we conclude that even though both components have the same expected performance we can still have $I_{N B}^{*(1)}(t)>I_{N B}^{*(2)}(t)$

\section{Importance measures for semi-Markov processes}

In the previous sections we assumed that each life cycle of a component was deterministic with respect to the states the component transited through. As a result the next and previous states at a given point of time, denoted respectively $X_{i}^{+}(t)$ and $X_{i}^{-}(t)$ were both determined with probability one by the current state $X_{i}(t)$. We now relax this assumption, and allow the components to follow a general semi-Markov process, where the state transitions follow a Markov chain, referred to as the built-in Markov chain. Thus, each time component $i$ enters a state $u \in S_{i}$, it remains there for a random amount of time, and then makes a transition into state $v \in S_{i}$ with probability $P_{u v}^{(i)}$. The full matrix of transition probabilities for the built-in Markov chain for component $i$ is denoted $\boldsymbol{P}^{(i)}, i \in C$. Given this matrix we have that:

$$
P\left(X_{i}^{+}(t)=v \mid X_{i}(t)=u\right)=P_{u v}^{(i)}, \quad u, v \in S_{i} .
$$

In order to find a similar expression for the conditional distribution of $X_{i}^{-}(t)$, we need the transition matrix for the backwards version of the built-in Markov chain, which we denote by $\boldsymbol{Q}^{(i)}$. It then follows that:

$$
P\left(X_{i}^{-}(t)=v \mid X_{i}(t)=u\right)=Q_{u v}^{(i)}, \quad u, v \in S_{i} .
$$

Within this more general context the definitions of $I_{N B}^{(i)}(t)$ and $I_{P B}^{(i)}(t)$ given in Eq. (3.3) and Eq. (3.4) are still valid. However, the equations Eq. (3.5) and Eq. (3.6) have to be modified as follows:

$$
\begin{aligned}
& I_{N B}^{(i)}(t)=\sum_{u, v \in S_{i}} P[\phi(u, \boldsymbol{X}(t)) \neq \phi(v, \boldsymbol{X}(t))] \cdot P\left[X_{i}(t)=u\right] \cdot P_{u v}^{(i)} \\
& I_{P B}^{(i)}(t)=\sum_{u, v \in S_{i}} P[\phi(u, \boldsymbol{X}(t)) \neq \phi(v, \boldsymbol{X}(t))] \cdot P\left[X_{i}(t)=u\right] \cdot Q_{u v}^{(i)}
\end{aligned}
$$


Note that the measures $I_{N B}^{(i)}(t)$ and $I_{P B}^{(i)}(t)$ defined respectively in Eq. (4.3) and Eq. (4.4) are essentially the same measures as we introduced previously in respectively Eq. (3.3) and Eq. (3.4). The only difference is that the mathematical expressions now must to take into account that a more general probability model is used for the component processes.

We henceforth focus on the asymptotic properties of the processes and ommit the time $t$ from the notation. For component $i \in C$ we denote the stationary probabilities of the built-in Markov chain by $\pi_{u}^{(i)}, u \in S_{i}$. We then have the following well-known relation between the transition matrices $\boldsymbol{P}^{(i)}$ and $\boldsymbol{Q}^{(i)}$ (see e.g., Ross (2014)):

$$
Q_{u v}^{(i)}=\frac{\pi_{v}^{(i)}}{\pi_{u}^{(i)}} P_{v u}^{(i)}, \quad u, v \in S_{i} .
$$

Note that if the stationary distribution of the built-in Markov chain is uniform, i.e., if $\pi_{u}^{(i)}=1 /\left(r_{i}+1\right)$, for all $u \in S_{i}$, we have:

$$
\boldsymbol{Q}^{(i)}=\left(\boldsymbol{P}^{(i)}\right)^{T}, \quad i \in C .
$$

It is well-known that an irreducible aperiodic finite Markov chain has a uniform stationary distribution if and only if $\boldsymbol{P}^{(i)}$ is a doubly stochastic matrix, i.e., all row sums and column sums are equal to 1.

In order to proceed we now introduce the times spent in each state between the transitions. More specifically, we let:

$$
W_{k u}^{(i)}=\text { The } k \text { th waiting time in state } u \text { for component } i .
$$

We assume that all the waiting times are independent, and that for all components $i \in C$ and states $u \in S_{i}$ the waiting times $W_{1 u}^{(i)}, W_{2 u}^{(i)}, \ldots$ are identically distributed with finite mean $\mu_{u}^{(i)}$. Then it follows from standard renewal theory (see e.g., Ross (2014)) that the stationary distribution of $X_{i}$ is given by:

$$
P\left[X_{i}=u\right]=\frac{\pi_{u}^{(i)} \mu_{u}^{(i)}}{\sum_{v \in S_{i}} \pi_{v}^{(i)} \mu_{v}^{(i)}}, \quad u \in S_{i}, i \in C .
$$

Combining Eq. (4.6) with Eq. (4.3) and Eq. (4.4) we get the following expressions for the stationary importance measures:

$$
\begin{aligned}
& I_{N B}^{(i)}=\sum_{u, v \in S_{i}} P[\phi(u, \boldsymbol{X}) \neq \phi(v, \boldsymbol{X})] \cdot \frac{\pi_{u}^{(i)} \mu_{u}^{(i)}}{\sum_{v \in S_{i}} \pi_{v}^{(i)} \mu_{v}^{(i)}} \cdot P_{u v}^{(i)} \\
& I_{P B}^{(i)}=\sum_{u, v \in S_{i}} P[\phi(u, \boldsymbol{X}) \neq \phi(v, \boldsymbol{X})] \cdot \frac{\pi_{u}^{(i)} \mu_{u}^{(i)}}{\sum_{v \in S_{i}} \pi_{v}^{(i)} \mu_{v}^{(i)}} \cdot Q_{u v}^{(i)}
\end{aligned}
$$

Using these formulas it is easy to establish a sufficient condition for when $I_{N B}^{(i)}=I_{P B}^{(i)}$.

Theorem 4.1 Assume that $\mu_{0}^{(i)}=\cdots=\mu_{r_{i}}^{(i)}$. Then $I_{N B}^{(i)}=I_{P B}^{(i)}$. 
Proof: If $\mu_{0}^{(i)}=\cdots=\mu_{r_{i}}^{(i)}$, the stationary distribution given in Eq. (4.6) is simplified to:

$$
P\left[X_{i}=u\right]=\pi_{u}^{(i)}, \quad u \in S_{i}, i \in C .
$$

Inserting this into Eq. (4.7) and Eq. (4.8), we get:

$$
\begin{gathered}
I_{N B}^{(i)}=\sum_{u, v \in S_{i}} P[\phi(u, \boldsymbol{X}) \neq \phi(v, \boldsymbol{X})] \cdot \pi_{u}^{(i)} \cdot P_{u v}^{(i)} \\
I_{P B}^{(i)}=\sum_{u, v \in S_{i}} P[\phi(u, \boldsymbol{X}) \neq \phi(v, \boldsymbol{X})] \cdot \pi_{u}^{(i)} \cdot Q_{u v}^{(i)}
\end{gathered}
$$

We then insert the expression for $Q_{u v}^{(i)}$ given in Eq. (4.5) into the last equation and get:

$$
I_{P B}^{(i)}=\sum_{u, v \in S_{i}} P[\phi(u, \boldsymbol{X}) \neq \phi(v, \boldsymbol{X})] \cdot \pi_{v}^{(i)} \cdot P_{v u}^{(i)}
$$

Hence, by interchanging $u$ and $v$ we get that $I_{N B}^{(i)}=I_{P B}^{(i)}$ as stated

Another special case occurs when the transition matrix $\boldsymbol{P}^{(i)}$ is doubly stochastic.

Theorem 4.2 Assume that the transition matrix $\boldsymbol{P}^{(i)}$ is doubly stochastic. Then we have:

$$
\begin{aligned}
I_{N B}^{(i)} & =\sum_{u, v \in S_{i}} P[\phi(u, \boldsymbol{X}) \neq \phi(v, \boldsymbol{X})] \cdot \frac{\mu_{u}^{(i)}}{\sum_{v \in S_{i}} \mu_{v}^{(i)}} \cdot P_{u v}^{(i)} \\
I_{P B}^{(i)} & =\sum_{u, v \in S_{i}} P[\phi(u, \boldsymbol{X}) \neq \phi(v, \boldsymbol{X})] \cdot \frac{\mu_{u}^{(i)}}{\sum_{v \in S_{i}} \mu_{v}^{(i)}} \cdot P_{v u}^{(i)}
\end{aligned}
$$

Proof: If the transition matrix $\boldsymbol{P}^{(i)}$ is doubly stochastic, it is easy to see that the stationary distribution of the built-in Markov chain is uniform. Hence, the stationary distribution given in Eq. (4.6) is simplified to:

$$
P\left[X_{i}=u\right]=\frac{\mu_{u}^{(i)}}{\sum_{v \in S_{i}} \mu_{v}^{(i)}}, \quad u \in S_{i}, i \in C .
$$

Moreover, the transition matrix $\boldsymbol{Q}^{(i)}$ is equal to $\left(\boldsymbol{P}^{(i)}\right)^{T}$. That is:

$$
Q_{u v}^{(i)}=P_{v u}^{(i)}, \quad \text { for all } u, v \in S_{i} .
$$

By combining these observations the stated result follows

We observe that by Eq. (4.7) and Eq. (4.8) $I_{N B}^{(i)}$ and $I_{P B}^{(i)}$ depends both on the stationary distributions and the transition matrices $\boldsymbol{P}^{(i)}$ and $\boldsymbol{Q}^{(i)}$. Thus, even if two components have equal stationary distributions, they may still have different importance. The following example illustrates this.

Example 4.3 We consider once again a multistate system $(C, \phi)$ where $C=\{1,2\}$, and where both components have three possible states, $0,1,2$. For simplicity we again let $f_{i}(u)=$ $u, u=0,1,2, i=1,2$. Moreover, as before the structure function is given by:

$$
\phi\left(X_{1}(t), X_{2}(t)\right)=\min \left(f_{1}\left(X_{1}(t)\right), f_{2}\left(X_{2}(t)\right)\right) .
$$


The transition matrices of the built-in Markov chains are:

$$
\boldsymbol{P}^{(1)}=\left[\begin{array}{lll}
0.1, & 0.3, & 0.6 \\
0.6, & 0.1, & 0.3 \\
0.3, & 0.6, & 0.1
\end{array}\right], \quad \boldsymbol{P}^{(2)}=\left[\begin{array}{lll}
0.7, & 0.1, & 0.2 \\
0.2, & 0.7, & 0.1 \\
0.1, & 0.2, & 0.7
\end{array}\right],
$$

while the mean waiting times are:

$$
\mu_{0}^{(i)}=2.5, \quad \mu_{1}^{(i)}=3.5, \quad \mu_{2}^{(i)}=4.0, \quad i=1,2 .
$$

It is easy to see that both $\boldsymbol{P}^{(1)}$ and $\boldsymbol{P}^{(2)}$ are doubly stochastic, implying that the stationary distributions of the built-in Markov chains are uniform. Hence, we may calculate importance using Theorem 4.2. In particular, the stationary distributions can be calculated using the simplified formula given in Eq. (4.9), and we get:

$$
P\left[X_{i}=0\right]=0.25, \quad P\left[X_{i}=1\right]=0.35, \quad P\left[X_{i}=2\right]=0.40, \quad i=1,2 .
$$

We now focus on $I_{N B}^{(i)}$. The corresponding results for $I_{P B}^{(i)}$ are obtained in a similar fashion and are approximately the same in this case. In order to calculate $I_{N B}^{(i)}$, we need to compute a sum over all $u, v \in S_{i}$. Since, however, we obviously have:

$$
P\left[\phi\left(u, X_{2}\right) \neq \phi\left(v, X_{2}\right)\right]=0 \quad \text { if } u=v,
$$

only the terms where $u \neq v$ need to be included. Moreover, by symmetry we of course also have:

$$
P\left[\phi\left(u, X_{2}\right) \neq \phi\left(v, X_{2}\right)\right]=P\left[\phi\left(v, X_{2}\right) \neq \phi\left(u, X_{2}\right)\right] .
$$

Using this we get the following non-zero probabilities for component 1 :

$$
\begin{aligned}
& P\left[\phi\left(0, X_{2}\right) \neq \phi\left(1, X_{2}\right)\right]=P\left[X_{2}=1\right]+P\left[X_{2}=2\right]=0.75, \\
& P\left[\phi\left(0, X_{2}\right) \neq \phi\left(2, X_{2}\right)\right]=P\left[X_{2}=1\right]+P\left[X_{2}=2\right]=0.75, \\
& P\left[\phi\left(1, X_{2}\right) \neq \phi\left(0, X_{2}\right)\right]=P\left[\phi\left(0, X_{2}\right) \neq \phi\left(1, X_{2}\right)\right]=0.75, \\
& P\left[\phi\left(1, X_{2}\right) \neq \phi\left(2, X_{2}\right)\right]=P\left[X_{2}=2\right]=0.40, \\
& P\left[\phi\left(2, X_{2}\right) \neq \phi\left(0, X_{2}\right)\right]=P\left[\phi\left(0, X_{2}\right) \neq \phi\left(2, X_{2}\right)\right]=0.75, \\
& P\left[\phi\left(2, X_{2}\right) \neq \phi\left(1, X_{2}\right)\right]=P\left[\phi\left(1, X_{2}\right) \neq \phi\left(2, X_{2}\right)\right]=0.40 .
\end{aligned}
$$

Moreover, since these probabilities only depend on the stationary distribution of component 2 , and both components have the same stationary distribution, we get exactly the same probabilities for component 2 . Thus, we have all the quantities needed in order to compute the importance measures using the formula for $I_{N B}^{(i)}$ given in Theorem 4.2, and we get:

$$
I_{N B}^{(1)}=0.55425, \quad I_{N B}^{(2)}=0.18475
$$

We observe that component 1 is much more important than component 2, even though they both have exactly the same stationary distributions. The reason for this is that component 2 has a much higher probability of staying in the same state when a transition happens compared to component 1, i.e., 0.7 versus 0.1 . Hence, for component 2 most of the weight from the transition probabilities are put on terms where $u=v$, and for these terms $P\left[\phi\left(X_{1}, u\right) \neq \phi\left(X_{1}, v\right)\right]=0$. For component 1 on the other hand most of 
the weight from the transition probabilities are put on terms where $u \neq v$, and for these terms $P\left[\phi\left(u, X_{2}\right) \neq \phi\left(v, X_{2}\right)\right]>0$.

In many applications transitions from one state directly back to the same state does not make sense. In order to avoid this we may e.g., replace the matrices of transition probabilities of the built-in Markov chains with the following:

$$
\boldsymbol{P}^{(1)}=\left[\begin{array}{lll}
0.0, & 0.1, & 0.9 \\
0.9, & 0.0, & 0.1 \\
0.1, & 0.9, & 0.0
\end{array}\right], \quad \boldsymbol{P}^{(2)}=\left[\begin{array}{ccc}
0.0, & 0.9, & 0.1 \\
0.1, & 0.0, & 0.9 \\
0.9, & 0.1, & 0.0
\end{array}\right],
$$

We observe that in these transition matrices all the diagonal terms are 0.0. Thus, the probability of staying in the same state after a transition has happened is zero.

It is again easy to verify that both $\boldsymbol{P}^{(1)}$ and $\boldsymbol{P}^{(2)}$ are doubly stochastic. This implies that the stationary probabilities are the same as in the previous case. The change in transition probabilities, however, implies that the importance measures change considerably. In this case we get:

$$
I_{N B}^{(1)}=0.61175, \quad I_{N B}^{(2)}=0.62575,
$$

implying that component 2 is now slightly more important than component 1.

By comparing transition matrices we also notice that $\boldsymbol{P}^{(1)}=\left(\boldsymbol{P}^{(2)}\right)^{T}$. By Theorem 4.2 this implies that:

$$
I_{P B}^{(1)}=I_{N B}^{(2)}, \quad \text { and } \quad I_{P B}^{(2)}=I_{N B}^{(1)} .
$$

Hence, the importance ranking is reversed if we apply the p-Birnbaum measure instead of the n-Birnbaum measure

\subsection{Expected physical criticality for semi-Markov processes}

We close this section by briefly showing how the importance measures based on expected physical criticality, introduced in Subsection 3.1 can be extended to semi-Markov processes. We observe that Eq. (3.8) and Eq. (3.9) are valid also in the general case. In order to calculate the $n^{*}$-Birnbaum measure and the $p^{*}$-Birnbaum measure, we again expand Eq. (3.8) and Eq. (3.9) by conditioning on the state of component $i$ and get formulas similar to Eq. (4.3) and Eq. (4.4):

$$
\begin{aligned}
& I_{N B}^{*(i)}(t)=\sum_{u, v \in S_{i}} E|\phi(u, \boldsymbol{X}(t))-\phi(v, \boldsymbol{X}(t))| \cdot P\left[X_{i}(t)=u\right] \cdot P_{u v}^{(i)} \\
& \left.I_{P B}^{*(i)}(t)=\sum_{u, v \in S_{i}} E \mid \phi(u, \boldsymbol{X}(t))-\phi(v, \boldsymbol{X}(t))\right] \cdot P\left[X_{i}(t)=u \mid \cdot Q_{u v}^{(i)}\right.
\end{aligned}
$$

Focussing on the asymptotic properties and using the same arguments as we $\operatorname{did}$ for $I_{N B}^{(i)}$ and $I_{P B}^{(i)}$, we get the following analogues to Eq. (4.7) and Eq. (4.8):

$$
\begin{aligned}
I_{N B}^{*(i)} & =\sum_{u, v \in S_{i}} E|\phi(u, \boldsymbol{X})-\phi(v, \boldsymbol{X})| \cdot \frac{\pi_{u}^{(i)} \mu_{u}^{(i)}}{\sum_{v \in S_{i}} \pi_{v}^{(i)} \mu_{v}^{(i)}} \cdot P_{u v}^{(i)} \\
I_{P B}^{*(i)} & =\sum_{u, v \in S_{i}} E|\phi(u, \boldsymbol{X})-\phi(v, \boldsymbol{X})| \cdot \frac{\pi_{u}^{(i)} \mu_{u}^{(i)}}{\sum_{v \in S_{i}} \pi_{v}^{(i)} \mu_{v}^{(i)}} \cdot Q_{u v}^{(i)}
\end{aligned}
$$


Having these formulas the following results can easily be proved using exactly the same arguments as we used for the corresponding results for $I_{N B}^{(i)}$ and $I_{P B}^{(i)}$ :

Theorem 4.4 Assume that $\mu_{0}^{(i)}=\cdots=\mu_{r_{i}}^{(i)}$. Then $I_{N B}^{*(i)}=I_{P B}^{*(i)}$.

Theorem 4.5 Assume that the transition matrix $\boldsymbol{P}^{(i)}$ is doubly stochastic. Then we have:

$$
\begin{aligned}
& I_{N B}^{*(i)}=\sum_{u, v \in S_{i}} E|\phi(u, \boldsymbol{X})-\phi(v, \boldsymbol{X})| \cdot \frac{\mu_{u}^{(i)}}{\sum_{v \in S_{i}} \mu_{v}^{(i)}} \cdot P_{u v}^{(i)} \\
& I_{P B}^{*(i)}=\sum_{u, v \in S_{i}} E|\phi(u, \boldsymbol{X})-\phi(v, \boldsymbol{X})| \cdot \frac{\mu_{u}^{(i)}}{\sum_{v \in S_{i}} \mu_{v}^{(i)}} \cdot P_{v u}^{(i)}
\end{aligned}
$$

We close this section by considering an example.

Example 4.6 We consider the multistate system $(C, \phi)$ introduced in Example 4.3. In order to compute $I_{N B}^{*(1)}$ we start out by determining $E\left|\phi\left(u, X_{2}\right)-\phi\left(v, X_{2}\right)\right|$ for all $u, v \in S_{1}$. We note again that:

$$
E\left|\phi\left(u, X_{2}\right)-\phi\left(v, X_{2}\right)\right|=0 \quad \text { if } u=v,
$$

implying that only the terms where $u \neq v$ need to be included. Moreover, by symmetry we have:

$$
E\left|\phi\left(u, X_{2}\right)-\phi\left(v, X_{2}\right)\right|=E\left|\phi\left(v, X_{2}\right)-\phi\left(u, X_{2}\right)\right| .
$$

Using this we get the following non-zero expectations for component 1 :

$$
\begin{aligned}
& E\left|\phi\left(0, X_{2}\right)-\phi\left(1, X_{2}\right)\right|=1 \cdot P\left[X_{2}=1\right]+1 \cdot P\left[X_{2}=2\right]=0.75, \\
& E\left|\phi\left(0, X_{2}\right)-\phi\left(2, X_{2}\right)\right|=1 \cdot P\left[X_{2}=1\right]+2 \cdot P\left[X_{2}=2\right]=1.15, \\
& E\left|\phi\left(1, X_{2}\right)-\phi\left(0, X_{2}\right)\right|=E\left|\phi\left(0, X_{2}\right)-\phi\left(1, X_{2}\right)\right|=0.75, \\
& E\left|\phi\left(1, X_{2}\right)-\phi\left(2, X_{2}\right)\right|=1 \cdot P\left[X_{2}=2\right]=0.40, \\
& E\left|\phi\left(2, X_{2}\right)-\phi\left(0, X_{2}\right)\right|=E\left|\phi\left(0, X_{2}\right)-\phi\left(2, X_{2}\right)\right|=1.15, \\
& E\left|\phi\left(2, X_{2}\right)-\phi\left(1, X_{2}\right)\right|=E\left|\phi\left(1, X_{2}\right)-\phi\left(2, X_{2}\right)\right|=0.40 .
\end{aligned}
$$

Moreover, since these expectations only depend on the stationary distribution of component 2 , and both components have the same stationary distribution, we get exactly the same expectations for component 2 . Thus, we have all the quantities needed in order to compute the importance measures using the formula for $I_{N B}^{*(1)}$ and $I_{N B}^{*(2)}$ given in Theorem 4.5. If the transition matrices are:

$$
\boldsymbol{P}^{(1)}=\left[\begin{array}{lll}
0.1, & 0.3, & 0.6 \\
0.6, & 0.1, & 0.3 \\
0.3, & 0.6, & 0.1
\end{array}\right], \quad \boldsymbol{P}^{(2)}=\left[\begin{array}{lll}
0.7, & 0.1, & 0.2 \\
0.2, & 0.7, & 0.1 \\
0.1, & 0.2, & 0.7
\end{array}\right],
$$

we get that:

$$
I_{N B}^{*(1)}=0.66225, \quad I_{N B}^{*(2)}=0.22075
$$

In particular component 1 is much more important than component 2 , even though they both have exactly the same stationary distributions. If the transition matrices are:

$$
\boldsymbol{P}^{(1)}=\left[\begin{array}{lll}
0.0, & 0.1, & 0.9 \\
0.9, & 0.0, & 0.1 \\
0.1, & 0.9, & 0.0
\end{array}\right], \quad \boldsymbol{P}^{(2)}=\left[\begin{array}{ccc}
0.0, & 0.9, & 0.1 \\
0.1, & 0.0, & 0.9 \\
0.9, & 0.1, & 0.0
\end{array}\right],
$$


we get that:

$$
I_{N B}^{*(1)}=0.71775, \quad I_{N B}^{*(2)}=0.77975
$$

Since $\boldsymbol{P}^{(1)}=\left(\boldsymbol{P}^{(2)}\right)^{T}$, Theorem 4.5 implies that:

$$
I_{P B}^{*(1)}=I_{N B}^{*(2)}, \quad \text { and } \quad I_{P B}^{*(2)}=I_{N B}^{*(1)} .
$$

Hence, the importance ranking is reversed if we apply the $p^{*}$-Birnbaum measure instead of the $n^{*}$-Birnbaum measure.

We observe that these results are very similar to the corresponding results for $I_{N B}^{(1)}$ and $I_{N B}^{(2)}$ found in Example 4.3, except that the importance measures are somewhat greater in this case

\section{A numerical example}

The examples presented in the previous sections are of course extremely simple and carefully chosen in order to illustrate the theoretical results. For these examples it was very easy to calculate the importance measures manually. In this section we present a somewhat larger and more realistic example. It should be noted that it is not difficult to do the asymptotic calculations analytically. Still in many cases it is more convenient to estimate measures using Monte Carlo simulation. In a simulation study it is also possible to estimate nonasymptotic results as well. For a description of the discrete event simulation procedure we have applied, see Huseby and Natvig (2010) and Huseby and Natvig (2012). An application of the methodology is given in Natvig et al. (2009).

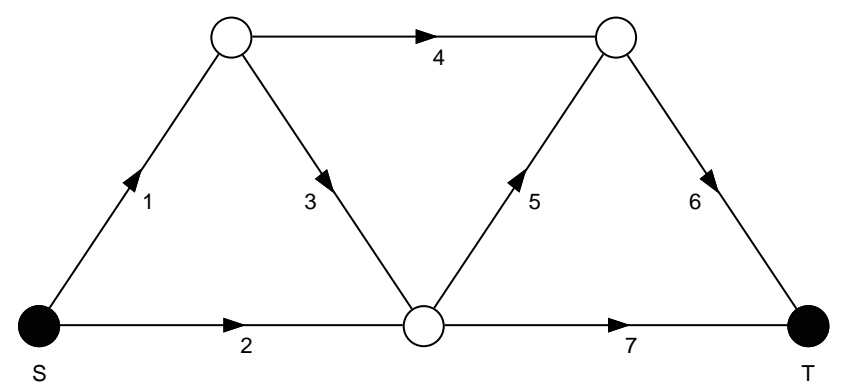

Figure 5.1: A network flow system

In this example we consider a directed network flow system consisting of 7 components representing the directed edges of the network. For an extensive introduction to network flow systems and reliability we recommend Todinov (2013). The system is illustrated in Figure 5.1. The physical state functions of the components, $f_{1}, \ldots, f_{7}$, represent the flow capacity of the components. The physical state of the system is the amount of flow that can be sent trough the network from the source node $S$ to the terminal node $T$.

In order to express the system state as a function of the component states we identify the minimal cut sets in the network. These are $K_{1}=\{1,2\}, K_{2}=\{1,5,7\}, K_{3}=\{2,3,4\}$, $K_{4}=\{4,5,7\}, K_{5}=\{2,3,6\}, K_{6}=\{6,7\}$. According to the well-known max-flow-min cut 
theorem (see Ford and Fulkerson (1956)) we then have ${ }^{1}$ :

$$
\phi(\boldsymbol{X}(t))=\min _{1 \leq j \leq 6} \sum_{i \in K_{j}} f_{i}\left(X_{i}(t)\right) .
$$

The component state functions in this example are given by:

$$
\begin{aligned}
& f_{1}(u)=f_{6}(u)=2.5 \cdot u, \quad u=0,1,2 . \\
& f_{2}(u)=f_{7}(u)=1.5 \cdot u, \quad u=0,1,2 . \\
& f_{3}(u)=f_{5}(u)=5.0 \cdot u, \quad u=0,1 . \\
& f_{4}(u)=1.0 \cdot I(u=1)+2.5 \cdot I(u=2), \quad u=0,1,2 .
\end{aligned}
$$

We observe that if all components are in their respective top state, the maximal flow through the network is 8 . The mean waiting times for the different states of the components are given in Table 5.1 .

\begin{tabular}{c|ccc} 
Comp. & $\mu_{0}^{(i)}$ & $\mu_{1}^{(i)}$ & $\mu_{2}^{(i)}$ \\
\hline 1 & 2.5 & 3.5 & 4.5 \\
2 & 2.5 & 3.5 & 4.5 \\
3 & 4.5 & 5.5 & - \\
4 & 2.5 & 3.5 & 4.5 \\
5 & 4.5 & 5.5 & - \\
6 & 2.5 & 3.5 & 4.5 \\
7 & 2.5 & 3.5 & 4.5 \\
\hline
\end{tabular}

Table 5.1: Mean waiting times

Finally, the transition probabilities for the components are:

$$
\begin{gathered}
\boldsymbol{P}^{(1)}=\boldsymbol{P}^{(2)}=\boldsymbol{P}^{(6)}=\boldsymbol{P}^{(7)}=\left[\begin{array}{lll}
0.0, & 0.0, & 1.0 \\
1.0, & 0.0, & 0.0 \\
0.4, & 0.6, & 0.0
\end{array}\right], \\
\boldsymbol{P}^{(3)}=\boldsymbol{P}^{(5)}=\left[\begin{array}{ll}
0.0, & 1.0 \\
1.0, & 0.0
\end{array}\right], \quad \boldsymbol{P}^{(4)}=\left[\begin{array}{lll}
0.0, & 0.0, & 1.0 \\
1.0, & 0.0, & 0.0 \\
0.6, & 0.4, & 0.0
\end{array}\right] .
\end{gathered}
$$

In the simulation all waiting times were sampled from exponential distributions with the respective mean values. For the asymptotic results, however, the choice of distribution is of course not important.

We ran 10000 simulations of the system over a time frame of 500 time units. The resulting estimated importance measures are given in Table 5.2.

Due to the structural symmetry in the network as well as the chosen distribution parameters and transition probabilities, the components are grouped into four sets: $C_{1}=\{1,6\}$, $C_{2}=\{2,7\}, C_{3}=\{3,5\}, C_{4}=\{4\}$ with respect to importance. Within these groups the

\footnotetext{
${ }^{1}$ It should be noted that there exist efficient algorithms for calculating the system state without having to identify all the minimal cut sets. See e.g., Dinic (1970) where an algorithm of order $O\left(v^{2} \cdot n\right)$ is presented, and where $v$ and $n$ denote respectively the number of nodes and edges in the network. However, in the simple case considered here with only 6 minimal cut sets, we just use the standard formula.
} 


\begin{tabular}{c|llll} 
Comp. & $I_{N B}^{(i)}$ & $I_{P B}^{(i)}$ & $I_{N B}^{*(i)}$ & $I_{P B}^{*(i)}$ \\
\hline 1 & 0.53 & 0.56 & 1.34 & 1.48 \\
2 & 0.64 & 0.66 & 1.24 & 1.39 \\
3 & 0.34 & 0.34 & 0.83 & 0.83 \\
4 & 0.40 & 0.40 & 0.77 & 0.83 \\
5 & 0.34 & 0.34 & 0.83 & 0.83 \\
6 & 0.53 & 0.56 & 1.34 & 1.48 \\
7 & 0.64 & 0.66 & 1.24 & 1.39 \\
\hline
\end{tabular}

Table 5.2: Estimated importance

importance measures are equal regardless of which measure we use. However, we observe that the overall ranking depends on the chosen importance measure. If we choose $I_{N B}^{(i)}$ or $I_{P B}^{(i)}$, the components in $C_{2}$ are the most important components followed by $C_{1}, C_{4}$ and $C_{3}$. On the other hand if we choose $I_{N B}^{*(i)}$, the components in $C_{1}$ are the most important components followed by $C_{2}, C_{3}$ and $C_{4}$. Finally, if we choose $I_{P B}^{*(i)}$, the components in $C_{1}$ are still the most important components followed by $C_{2}$. In this case, the components in $C_{3}$ and $C_{4}$ are tied.

This example shows that there is a difference between just being critical and the physical effect of being critical. The components in $C_{2}$ have a very high probability of being critical to the system. However, the components in this set have lower flow capacities compared to the components in $C_{1}$. Thus, the ranking of these two sets is reversed when the measures $I_{N B}^{*(i)}$ or $I_{P B}^{*(i)}$ are used. The same effect can be seen in the ranking between $C_{3}$ and $C_{4}$.

The suggested measures allow the analyst to examine criticality from different viewpoints which is of interest both in a diagnostic setting as well as when the analysis is done in order to support decisions regarding improvement of the system.

\section{Conclusions}

In the present paper we have described a framework for modelling repairable multistate systems emphasising the physical properties of the components and the system. Within this framework we have generalised the Birnbaum measure to such systems in four different ways. Two of the suggested measures define component criticality at a given point of time relative to the next state of the component. These measures have a forward-looking focus which is often useful when the analysis is done in order to support decisions regarding improvement of the system. The two other measures define component criticality at a given point of time relative to the previous state of the component. These measures have a backward-looking focus which is often useful in a diagnostic setting.

The suggested measures can also be classified with respect to how criticality is measured. Two of the suggested measures focus on whether a component is critical or not, while the other two measures include information about the physical effects of being critical. Examples presented in the paper show that these viewpoints may result in different importance ranking of the components.

The paper considers both simple periodic component life cycles and more general semiMarkov processes and presents results on when the various measures are equal or can be simplified computationally. 
The proposed measures can be interpreted as time-dependent measures reflecting component importance at a given point of time. Alternatively, the measures can be interpreted as asymptotic measures representing component importance given that the system has reached a stationary status. In cases where the component life cycles are long compared to the time frame under consideration or when the component processes are not time-homogenous, it may be of interest to find non-asymptotic, time-independent importance measures. Future work within this area will focus on this problem as well as how the proposed measure can be utilised in a decision oriented context.

\section{Acknowledgements}

The authors would like to thank the reviewers for very helpful comments which lead to significant improvements of the paper.

\section{References}

Amrutkar, K. P. and K. K. Kamalja (2017). An overview of various importance measures of reliability system. International Journal of Mathematical, Engineering and Management Sciences 2(3), 150-171.

Barlow, R. E. and F. Proschan (1975). Importance of system components and fault tree events. Stochastic Proc. Appl. (3), 153-173.

Birnbaum, Z. W. (1969). On the importance of different components in a multicomponent system. In P. R. Krishnaia (Ed.), Multivariate Analysis - II, pp. 581-592. Academic Press, New York.

Borgonovo, E. and G. E. Apostolakis (2001). A new importance measure for risk-informed decision making. Reliability Engineering \& System Safety (72), 193-212.

Cai, Z., S. Si, Y. Liu, and J. Zhao (2018). Maintenance optimization of continuous state systems based on performance improvement. IEEE Transactions on Reliability $(67(2))$, $651-665$.

Cai, Z., S. Si, S. Sun, and C. Li (2016). Optimization of linear consecutive-k-out-of-n system with a birnbaum importance-based genetic algorithm. Reliability Engineering 83 System Safety (152), 248-258.

Dinic, E. A. (1970). Algorithm for solution of a problem of maximum flow in a network with power estimation. Sov. Math. Dokl. (11), 1277-1280.

Dui, H., S. Li, L. Xing, and H. Liu (2019). System performance-based joint importance analysis guided maintenance for repairable systems. Reliability Eng. E Sys. Safety (186), $162-175$.

Dui, H., S. Si, M. J. Zuo, and S. Sun (2015). Semi-markov process-based integrated importance measures for multi-state systems. IEEE Transactions on Reliability (64(2)), $754-765$. 
Dui, H., C. Zhang, and X. Zheng (2020). Component joint importance measures for maintenances in submarine blowout preventer system. J. of Loss Prevention in the Proc. Indust. (63), 1-10.

Ford, L. R. and D. R. Fulkerson (1956). Maximal flow through a network. Canadian Journ. Math. (8), 399-404.

Fussell, J. B. and W. E. Vesely (1972). A new methodology for obtaining cut sets for fault trees. Trans. of the Amer. Nuclear Soc. (15), 262-263.

Griffith, W. (1980). Multi-state reliability models. Journal of Applied Probability (17), 735-744.

Hosseini, S., K. Barker, and J. E. Ramirez-Marquez (2016). A review of definitions and measures of system resilience. Reliability Eng. \& Sys. Safety (145), 47-61.

Huseby, A. B. and B. Natvig (2010). Advanced discrete simulation methods applied to repairable multistate systems. In R. Bris, C. G. Soares, and S. Martorell (Eds.), Reliability, Risk and Safety. Theory and Applications, volume 1, pp. 659-666. London CRC Press.

Huseby, A. B. and B. Natvig (2012). Discrete event simulation methods applied to advanced importance measures of repairable components in multistate network flow systems. Reliability Eng. \&3 Sys. Safety (119), 186-198.

Levitin, G. and A. Lisnianski (1999). Importance and sensitivity analisis of multistate systems using the universal generating function. Reliability Eng. ES Sys. Safety (65), $271-282$.

Levitin, G., L. Podofillini, and E. Zio (2003). Generalised importance measures for multistate elements based on performance level restrictions. Reliability Eng. ES Sys. Safety (82), $287-298$.

Natvig, B. (1979). A suggestion for a new measure of importance of system components. Stochastic Processes and their Applications (9), 319-330.

Natvig, B. (1985). New light on measures of importance of system components. Scand. J. Statist (12), 43-54.

Natvig, B. (2011a). Measures of component importance in nonrepairable and repairable multistate strongly coherent systems. Methodol. Comput. Appl. Prob. (13), 523-547.

Natvig, B. (2011b). Multistate Systems Reliability Theory with Applications. New York, USA: John Wiley and Sons, Inc.

Natvig, B., K. A. Eide, J. Gåsemyr, A. B. Huseby, and S. L. Isaksen (2009). Simulation based analysis and an application to an offshore oil and gas production system of the Natvig measures of component importance in repairable systems. Reliability Engineering E System Safety (94), 1629-1638.

Natvig, B. and J. Gåsemyr (2009). New results on the Barlow-Proschan and Natvig measures of component importance in nonrepairable and repairable systems. Methodol. Comput. Appl. Prob. (11), 603-620. 
Natvig, B., A. B. Huseby, and M. Reistadbakk (2011). Measures of component importance in repairable multistate systems - a numerical study. Reliability Engineering \&s System Safety (96), 1680-1690.

Ramirez-Marquez, J. E. and D. W. Coit (2005). Composite importance measures for multistate systems with multistate components. IEEE Trans. Reliab. (54), 517-529.

Ramirez-Marquez, J. E. and D. W. Coit (2007). Multi-state component criticality analysis for reliability improvement in multi-state systems. Reliability Engineering \& System Safety (92), 1608-1619.

Ramirez-Marquez, J. E., C. M. Rocco, B. A. Gebre, D. W. Coit, and M. Tortorella (2006). New insights on multi-state component criticality and importance. Reliability Engineering E System Safety (91), 894-904.

Ross, S. (2014). Introduction to Probability Models - 11th Edition. Academic Press.

Si, S., H. Dui, Z. Cai, and S. Sun (2012). The integrated importance measure of multistate coherent systems for maintenance processes. IEEE Transaction on Reliability (61(2)), $266-273$.

Si, S., H. Dui, X. Zhao, S. Zhang, and S. Sun (2012). Integrated importance measure of component states based on loss of system performance. IEEE Transaction on Reliability (61(1)), 192-202.

Si, S., G. Levitin, H. Dui, and S. Sun (2013). Component state-based integrated importance measure for multi-state systems. Reliability Engineering 83 System Safety (116), 75-83.

Si, S., M. Liu, Z. Jiang, and T. Jin (2019). System reliability allocation and optimization based on generalized birnbaum importance measure. IEEE Transaction on Reliability $(68(3)), 831-843$.

Skutlaberg, K. and B. Natvig (2016). Minimization of the expected total net loss in a stationary multistate flow network system. Applied Mathematics (7), 793-817.

Todinov, M. T. (2013). Flow Networks. Elsevier Insights.

Wu, S., Y. Chen, Q. Wu, and Z. Wang (2016). Linking component importance to optimisation of preventive maintenance policy. Reliability Engineering 85 System Safety (146), $26-32$.

Wu, S. and F. Coolen (2013). A cost-based importance measure for system components: An extension of the birnbaum importance. European Journal of Operational Research (225), 189-195.

Zhu, X., Y. Fu, T. Yuan, and X. Wu (2017). Birnbaum importance based heuristics for multi-type component assignment problems. Reliability Engineering 83 System Safety (165), 209-221.

Zio, E., M. Marella, and L. Podofillini (2007). Importance measures-based prioritization for improving the performance of multi-state systems: application to the railway industry. Reliability Engineering \& System Safety (92), 1303-1314. 
Zio, E. and L. Podofillini (2003). Monte-carlo simulation analysis of the effects on different system performance levels on the importance on multistate components. Reliability Engineering $\& 3$ System Safety (82), 63-73.

Zio, E. and L. Podofillini (2006). Accounting for components interactions in the differential importance measure. Reliability Engineering $\&$ System Safety (91), 1163-1174.

Zio, E., L. Podofillini, and G. Levitin (2004). Estimation of the importance measures of multistate elements by monte carlo simulation. Reliability Engineering $\&$ System Safety (86), 191-204. 